\title{
Parallel central processing between tasks: Evidence from lateralized readiness potentials
}

\author{
Mei-Ching Lien \\ Oregon State University, Corvallis, Oregon \\ ERIC RUTHRUFF \\ University of New Mexico, Albuquerque, New Mexico \\ AND \\ Shulan Hsieh and Yen-Ting Yu \\ National Chung Cheng University, Min-Hsiung, Chia-Yi, Taiwan
}

\begin{abstract}
The present dual-task study used lateralized readiness potentials (LRPs) and behavioral measures to determine whether response activation for Task 1 and Task 2 can occur in parallel. We also examined whether task similarity (known as dimensional overlap) increases parallel central processing by making it difficult to selectively activate one task set. With dimensional overlap, the behavioral data replicated previous findings of backward correspondence effects: The Task 1 response was influenced by its compatibility with the Task 2 response. This finding suggests parallel response activation. The LRP data supported this conclusion: Task 2 response activation (indexed by the LRP) began before Task 1 central operations had finished. When there was no dimensional overlap, backward correspondence effects could not be measured, but the LRP data confirmed that parallel response activation still occurred. We argue that parallel response activation does occur, perhaps due to accidental activation of Task 2 mapping rules when the intention is to selectively execute Task 1 mapping rules.
\end{abstract}

When people attempt to perform two tasks at the same time, interference usually occurs. Although dual-task studies have generally yielded consistent findings, certain core issues have not yet been resolved. Recent debate has focused on whether central processes (activation and selection of responses) can operate in parallel for different tasks. Behavioral data (response time and accuracy) have provided useful clues, but no closure. What is needed is a more direct window into central operations as they emerge. The present study therefore addressed this issue by supplementing behavioral data with online electrophysiological measures.

\section{Overview of Previous Findings}

Previous dual-task studies have relied heavily on the psychological refractory period (PRP) paradigm, in which participants make speeded responses (R1 and R2) to stimuli for two different tasks ( $\mathrm{S} 1$ for Task 1 and S2 for Task 2). The key manipulation is the time between S1 and S2, known as the stimulus onset asynchrony (SOA). Typically, mean Task 1 response time (RT1) is constant across SOAs, but Task 2 response time (RT2) increases sharply at short SOAs (where the tasks are demanded nearly simultaneously). This RT2 increase is known as the PRP effect. To explain the PRP effect, Welford (1952) proposed that central processes can proceed for only one task at a time, resulting in a processing bottleneck (see Figure 1A).

Although early PRP studies generally supported the central bottleneck model (see Pashler, 1984), recent findings have raised doubt. One such finding is that although the Task 1 response is executed well before the Task 2 response, it is nevertheless modulated by correspondence with the Task 2 response. This effect of the Task 2 response on Task 1 performance is known as the backward correspondence effect. In Experiment 3 of Hommel (1998), for example, Task 1 was to press a left or right key in response to a red or green rectangle and Task 2 was to say "red" or "green" in response to the letter S or H. Hommel argued that if Task 2 central processes are completely postponed until Task 1 central processes are completed, then R2 should not be activated in time to influence RT1. Contrary to this prediction, RT1 at the short SOA was 28 msec faster when S1 and R2 corresponded (e.g., S1 was a red rectangle and R2 was the word "red") than when they did not. Backward correspondence effects have since been replicated using different tasks (Logan \& Schulkind, 2000) and different response modalities (Lien \& Proctor, 2000).

This backward correspondence effect led Hommel (1998) to propose that central operations contain two se- 

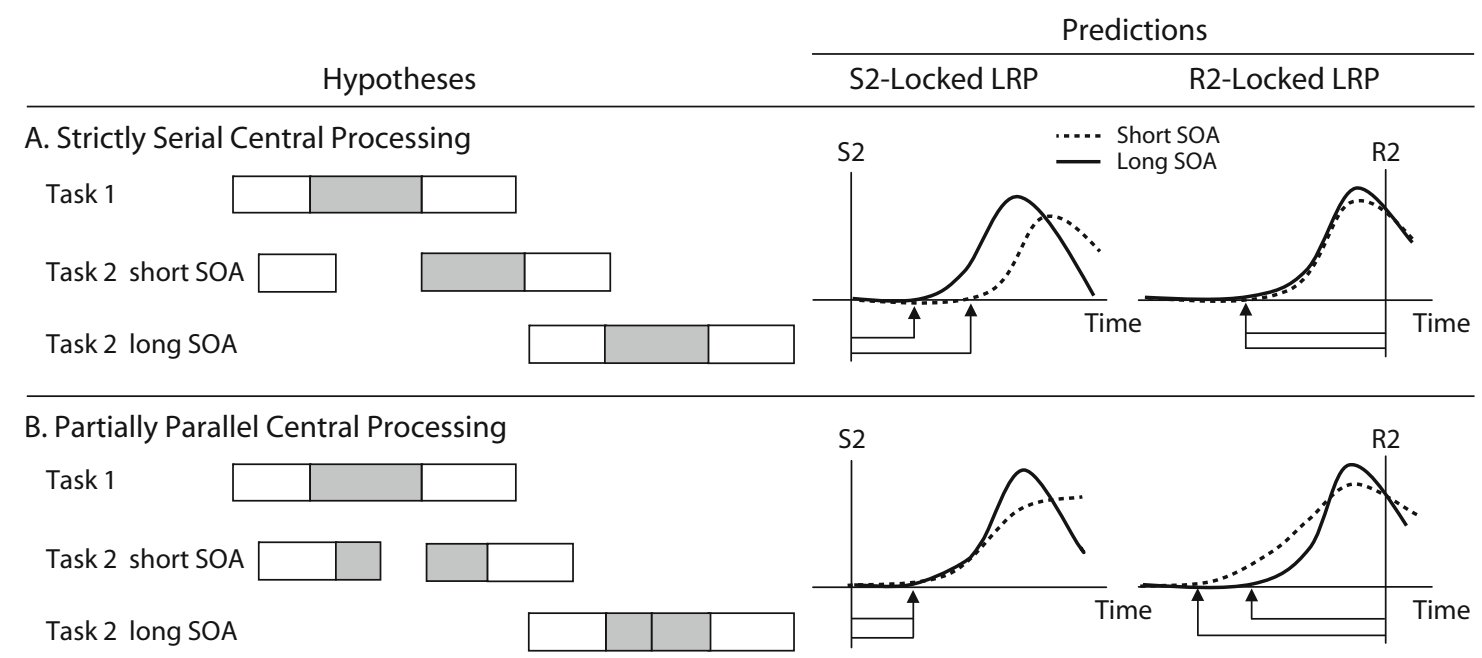

Figure 1. Two hypotheses regarding central processing in a psychological refractory period paradigm, along with their predictions for lateralized readiness potentials (LRPs) locked to the Task 2 stimulus (S2) and the Task 2 response (R2). Strictly serial central processing predicts that (1) the S2-locked LRP should arise earlier at the short stimulus onset asynchronies (SOAs) than at the long SOAs, whereas (2) the R2-locked LRP should arise at similar points in time, regardless of SOA. Partially parallel central processing predicts that (1) the S2-locked LRP should arise at similar points in time, regardless of SOA, whereas (2) the R2-locked LRP should arise earlier at the long SOA than at the short SOA. Central stages are shaded. In the Predictions column, the up-pointing arrows indicate LRP onset.

quential components (see Figure 1B). The first component, response activation (or stimulus-response translation), is not subject to the central bottleneck, but is too weak to support response initiation. The second component, response selection, is subject to the bottleneck. Hommel described this component as "a process that eventually drives a response above its selection threshold" (p. 1369, note 1).

\section{Limitations of the Backward Correspondence Paradigm}

Although backward correspondence studies are consistent with parallel central processing, they have limitations. First, measurement of backward correspondence effects requires dimensional overlap between the stimuli and/or responses for the two tasks (see Lien \& Proctor, 2002), defined as "the degree to which a stimulus and a response set ... are perceptually, structurally, or conceptually similar" (Kornblum \& Lee, 1995, p. 875). In Hommel's (1998) Experiment 3, for instance, the Task 1 stimulus (red/green) overlapped with the Task 2 response (red/green), which we henceforth refer to as $S 1-R 2$ dimensional overlap. In this case, the overlap is conceptual.

Dimensional overlap between tasks (i.e., task similarity) might make it difficult for participants to selectively activate one task set rather than the other. If dimensional overlap causes activation of one task to unintentionally activate the other task as well, then parallel central processing in the backward correspondence paradigm is a by-product of the conditions used to assess it (i.e., dimensional overlap). Accordingly, parallel central processing might not generalize to dual-task conditions without dimensional overlap, as in many real-world scenarios (e.g., talking while driving).
A further limitation is that backward correspondence effects merely suggest that some activation of the Task 2 response occurred at some point in time. It is difficult to say exactly when the response activation occurred or how strong it was. For instance, Task 2 response activation might be blocked $90 \%$ during Task 1 response selection, yet still produce a significant backward correspondence effect.

\section{Lateralized Readiness Potentials As an Index of Response Activation}

The lateralized readiness potential (LRP), a sensitive index of the degree of response preparation (see, e.g., Coles, 1989; Eimer, 1998), suggests an attractive alternative means of assessing parallel central processing. The LRP reflects the degree to which motor cortex is more active contralateral than ipsilateral to the correct response hand. The main question here is whether activation of the correct response hand for Task 2, as indexed by the LRP, begins while Task 1 central operations are still under way.

This LRP paradigm has several advantages over the backward correspondence paradigm. First, it can be used with or without dimensional overlap between tasks. Second, it provides a continuous measure of response activation, indicating not only how much response activation has occurred, but when it occurred.

Osman and Moore (1993) were the first to use this approach. They examined two LRP components in a PRP paradigm. The interval between S2 onset and LRP onset, called the S2-locked LRP interval, reflects the duration of processes prior to the onset of response preparation. The interval between LRP onset and R2 onset, called the $R 2$-locked LRP interval, reflects the duration of processes 
after the onset of response preparation. Osman and Moore reasoned that if Task 2 central operations are completely postponed at short SOAs due to a central bottleneck, then (1) the S2-locked LRP interval should increase as SOA decreases, and (2) the R2-locked LRP interval should be similar across SOAs. These predictions, illustrated in Figure 1A, were in fact confirmed (approximately) in two experiments. They concluded in favor of strictly serial central processing, contrary to backward correspondence studies.

Using a similar paradigm, Sangals, Roß, and Sommer (2004) reported a modest increase ( $\sim 40 \mathrm{msec})$ in the R2locked LRP interval at shorter SOAs (see also Sommer, Leuthold, \& Schubert, 2001). Rather than conclude that parallel central processing had occurred, they instead reached essentially the opposite conclusion. They argued that processing was limited not only by a central bottleneck but also by a "late" bottleneck at the response initiation stage. This conclusion might seem surprising, given the use of distinct response modalities (foot vs. manual); nevertheless, it is consistent with recent behavioral dualtask studies (Tombu \& Jolicœur, 2005).

In the present context, response conflicts are highly problematic. Response conflicts increase dual-task interference and perhaps deter parallel central processing (see Ruthruff, Johnston, \& Van Selst, 2001). Unfortunately, previous PRP-LRP studies have thus far used conditions likely to promote response conflicts. For example, Osman and Moore (1993) used two manual responses in Experiment 1 (see also Sommer et al., 2001), and used a foot response and a manual response in Experiment 2 (see also Sangals et al., 2004). To maximize the possibility of parallel central processing, the present study used modality combinations (vocal/manual) thought to minimize response conflicts.

\section{The Present Study}

Previous LRP studies have suggested strictly serial central processing, whereas backward correspondence studies have suggested partially parallel central processing. Although this state of affairs is perplexing, note that these approaches have not yet been utilized within the same study. Furthermore, both approaches have limitations. Backward correspondence studies provide useful clues, but use an indirect measure of response activation. In addition, the condition needed to assess parallel central processing - dimensional overlap between tasks - might actually cause the parallel central processing. LRP studies offer a tantalizingly direct measure of response activation, but have thus far been limited to conditions suspected to promote response conflicts, possibly deterring parallel central processing.

The present study aimed to resolve the controversy by using the best of both approaches. The design included a condition with dimensional overlap (which allowed the simultaneous measurement of LRPs and backward correspondence effects) and a condition without overlap (which allowed measurement of LRPs only). To manipulate S1-R2 dimensional overlap, we varied S1 while holding Task 2 constant (see Table 1). To minimize response conflicts, both conditions used a vocal Task 1 and a manual Task 2 . In addition to the S2-locked and R2-locked LRP analyses used in previous studies, we also time-locked Task 2 LRP to R1. As explained below, the R1-locked LRP analyses afford more definitive conclusions regarding parallel central processing.

\section{METHOD}

\section{Participants}

Ten participants (18-27 years of age) from the National Chung Cheng University were paid NT\$200 (approximately US\$7) per hour. All were native Chinese speakers with normal or correctedto-normal vision. Each participant performed two 2-h sessions, one each for the overlap and nonoverlap conditions (in separate laboratory visits). Session order was counterbalanced across participants.

\section{Apparatus and Stimuli}

S1 was the spoken word "beei" or "nan" (Chinese for north and south) in the nonoverlap condition and "tzuoo" or "yow" (Chinese for left and right) in the overlap condition (see Table 1). Each word was presented through speakers for $250 \mathrm{msec}$. The participants were to say "i" and "erh" (Chinese for one and two) in both conditions. These responses were chosen because they require minimal mouth movements, minimizing the electrical noise produced by muscle movements. Vocal RTs were collected using a microphone connected to an E-Prime response box. The experimenter entered the identity of the spoken responses. S2 was a red or green circle (3-cm diameter) in the screen center. The participants were to press the leftmost response box button with their left index finger for red circles or the rightmost button with their right index finger for green circles.

\section{Design and Procedure}

Each participant received one practice block of 64 trials followed by 15 regular blocks of 64 trials. On each trial, a fixation point was displayed for $800 \mathrm{msec}$, followed by the auditory S1 for $250 \mathrm{msec}$. After one of four SOAs $(50,150,600$, or 1,200 msec), the visual S2 was presented and remained visible until response. The participants were instructed to respond to both tasks quickly and accurately, but to emphasize Task 1 (to deter response grouping and reversal of the

Table 1

The Stimuli and Responses for Task 1 and Task 2 in the Nonoverlap Condition and the Overlap Condition

\begin{tabular}{|c|c|c|c|c|}
\hline \multirow[b]{2}{*}{ Condition } & \multicolumn{2}{|c|}{$\begin{array}{c}\text { Task } 1 \\
\text { (Auditory-Vocal Task) }\end{array}$} & \multicolumn{2}{|c|}{$\begin{array}{c}\text { Task } 2 \\
\text { (Visual-Manual Task) }\end{array}$} \\
\hline & Stimulus & Response & Stimulus & Response \\
\hline $\begin{array}{l}\text { Nonoverlap } \\
\text { Overlap }\end{array}$ & $\begin{array}{l}\text { "north"/"south" } \\
\text { "left"/“right" }\end{array}$ & $\begin{array}{l}\text { "one"/"two" } \\
\text { "one"/"two" }\end{array}$ & $\begin{array}{l}\text { red/green circle } \\
\text { red/green circle }\end{array}$ & $\begin{array}{l}\text { left/right keypress } \\
\text { left/right keypress }\end{array}$ \\
\hline
\end{tabular}

Note-The stimulus and response for Task 2 were constant in both conditions. The stimulusresponse mapping was arbitrary for both Task 1 and Task 2 in each condition. 
central processing order). RT and accuracy feedback were presented visually for $800 \mathrm{msec}$. The next trial began 1,200 msec later.

\section{Recording Equipment and Procedure}

Electroencephalographic (EEG) and electrooculographic (EOG) activity was recorded on each trial, using a Neuroscan $\mathrm{AgCl}$ 32channel Quik-Cap electrode cap (Compumedics, El Paso, TX). Horizontal EOG was recorded bipolarly from electrodes at the outer canthi of both eyes, and vertical EOG was recorded from electrodes above and below the midpoint of the right eye. All recording sites were referred to the linked mastoid. Electrode impedance was kept below $5 \mathrm{k} \Omega$. EEG and EOG were amplified using Neuroscan Syn$\mathrm{Amps}^{2}$ (Compumedics, El Paso, TX), filtered online with a bandpass of $0.05-50 \mathrm{~Hz}$, and digitized at $250 \mathrm{~Hz}$

\section{RESULTS}

\section{Behavioral Data Analyses}

Trials were excluded from the final analyses of RT and proportion of error (PE) if (1) either RT1 or RT2 was less than $100 \mathrm{msec}$ or greater than $3,000 \mathrm{msec}(0.7 \%$ of trials $)$ or (2) participants responded to Task 2 before Task 1 . In addition, trials were excluded from RT analyses if either response was incorrect or there were obvious eye movement artifacts. The resulting mean RTs are presented in Figure 2 and the mean PEs in Table 2.

The independent variables were task relationship (overlap and nonoverlap), correspondence (corresponding and noncorresponding), and $\operatorname{SOA}(50,150,600$, and $1,200 \mathrm{msec}$ ). Because task relationship and correspondence are not orthogonal (the nonoverlap condition has no correspondence manipulation), they cannot be included in the same crossed ANOVA. We therefore conducted two different ANOVAs. The main ANOVA examined the effects of task relationship and SOA (averaging across the corresponding and noncorresponding conditions). The correspondence ANOVA, conducted for the overlap condition, examined the effects of correspondence and SOA.

Task 1. The main ANOVA revealed no significant effects for RT1 or PE1. A roughly equal mean RT1 across
SOAs indicates that participants rarely (if ever) deferred Task 1 central operations.

The correspondence ANOVA on RT1 revealed a significant overall correspondence effect of $5 \mathrm{msec}[F(1,9)=$ $\left.5.34, M S_{\mathrm{e}}=1,555.84, p<.05\right]$. This effect interacted significantly with SOA $\left[F(3,27)=6.11, M S_{\mathrm{e}}=1,353.23\right.$, $p<.05]$; correspondence effects on RT1 were 18, 10, -5 , and $-2 \mathrm{msec}$ at the 50-, 150-, 600-, and 1,200-msec SOAs, respectively. Follow-up analyses showed that the correspondence effect was significant for both the 50- and 150 -msec SOAs $\left[F \mathrm{~s}(1,9) \geq 5.60, M S_{\mathrm{e}} \mathrm{s} \leq 2,161.60, p \mathrm{~s}<\right.$ .05], replicating earlier backward correspondence studies. For PE1, there was a trend toward more errors in the noncorresponding condition. However, neither the main effect of correspondence nor its interaction with SOA were significant $\left[F \mathrm{~s}(1,9) \leq 2.09, M S_{\mathrm{e}} \mathrm{s} \leq 0.0007, p \mathrm{~s} \geq .1818\right]$.

Task 2. For the main ANOVA on RT2, the main effect of SOA was significant $\left[F(3,27)=148.19, M S_{\mathrm{e}}=\right.$ $141,270.25, p<.0001]$, reflecting a 453-msec PRP effect. The PRP effects for the overlap (460 msec) and nonoverlap (439 msec) conditions did not differ significantly $[F(3,45)<1]$. For PE2, no effect was significant.

The correspondence ANOVA on RT2 revealed a significant 25-msec correspondence effect $[F(1,9)=16.52$, $\left.M S_{\mathrm{e}}=11,584.17, p<.01\right]$, which decreased as SOA increased $\left[F(3,27)=4.30, M S_{\mathrm{e}}=16,467.07, p<.05\right]$. PE2 was also affected by correspondence $[F(1,9)=6.79$, $\left.M S_{\mathrm{e}}=0.0032, p<.05\right]$ and its interaction with SOA $\left[F(3,27)=8.27, M S_{\mathrm{e}}=0.0022, p<.001\right]$.

\section{LRP Analyses}

LRP analyses were based on the same trials included in the RT analyses. LRP onset was estimated using the regression-based, single-subject one degree of freedom procedure recommended by Mordkoff and Gianaros (2000). This procedure requires fitting two lines, a preonset line and a postonset line, to the LRP data. The preonset line is constrained to be flat (slope $=0$ ), with a height

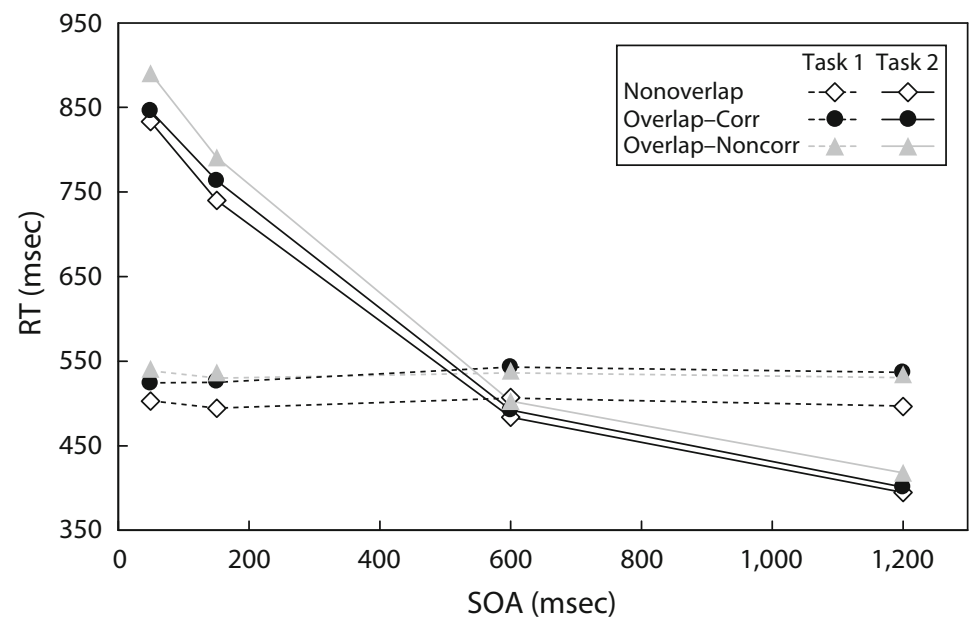

Figure 2. Mean response times (RTs) for Task 1 and Task 2 in the nonoverlap and overlap conditions as a function of stimulus onset asynchrony (SOA) and correspondence. Corr, corresponding; Noncorr, noncorresponding. 
Table 2

Proportions of Error for Task 1 and Task 2 in the Nonoverlap Condition and the Overlap Condition As a Function of Stimulus Onset Asynchrony (SOA) and Correspondence (Corresponding vs. Noncorresponding)

\begin{tabular}{|c|c|c|c|c|}
\hline \multirow[b]{2}{*}{ Condition } & \multicolumn{4}{|c|}{ SOA (msec) } \\
\hline & 50 & 150 & 600 & 1,200 \\
\hline \multicolumn{5}{|c|}{ Task 1} \\
\hline Nonoverlap & .017 & .011 & .006 & .005 \\
\hline \multicolumn{5}{|l|}{ Overlap } \\
\hline Corresponding & 011 & .012 & .008 & .007 \\
\hline Noncorresponding & .008 & .007 & .004 & .003 \\
\hline \multicolumn{5}{|c|}{ Task 2} \\
\hline Nonoverlap & .031 & .022 & .018 & .030 \\
\hline \multicolumn{5}{|l|}{ Overlap } \\
\hline Corresponding & .014 & .011 & .021 & .028 \\
\hline Noncorresponding & .032 & .023 & .037 & .044 \\
\hline
\end{tabular}

of 0 . The postonset line is constrained to pass through the peak of the LRP wave, but the slope is chosen to minimize the root-mean-squared difference between the fitted line and the LRP data. The estimated LRP onset is the time at which the preonset and postonset lines intersect. This method was applied separately to each participant's data for each condition. The mean LRP onset for each con- dition was determined by averaging the estimated LRP onsets across participants.

Because our main purpose was to compare LRP latencies between the overlap and nonoverlap conditions, we conducted only the main ANOVA (excluding the correspondence variable). For the overlap condition, we averaged the means for the correspondence and noncorrespondence conditions (weighting each condition equally).

S2-locked LRP. The data for each trial included a total EEG segment of 1,500 msec, from $200 \mathrm{msec}$ before S2 onset to $1,300 \mathrm{msec}$ after. S2-locked LRP waveforms were adjusted by subtracting the average voltage during a baseline interval from 200 to $10 \mathrm{msec}$ preceding S2 . We then measured LRPs by (1) calculating the difference waveforms between the $\mathrm{C}^{\prime}$ and $\mathrm{C}^{\prime}$ ' electrode sites for each trial, (2) sorting the trial data according to response hand (left vs. right) and then averaging, and (3) subtracting the average waveform for the right-hand trials from the average waveform for the left-hand trials. Thus,

$$
\mathrm{LPR}=\text { left hand }\left(\mathrm{C}^{\prime}{ }^{\prime}-\mathrm{C} 4^{\prime}\right)-\text { right hand }\left(\mathrm{C} 3^{\prime}-\mathrm{C} 4^{\prime}\right) \text {. }
$$

S2-locked LRP waveforms are shown in Figure 3, and the estimated LRP onset latencies are shown in Table 3. LRP onset latency increased as SOA decreased $[F(3,27)=$ $\left.116.99, M S_{\mathrm{e}}=668.66, p<.001\right]$. The delay in LRP
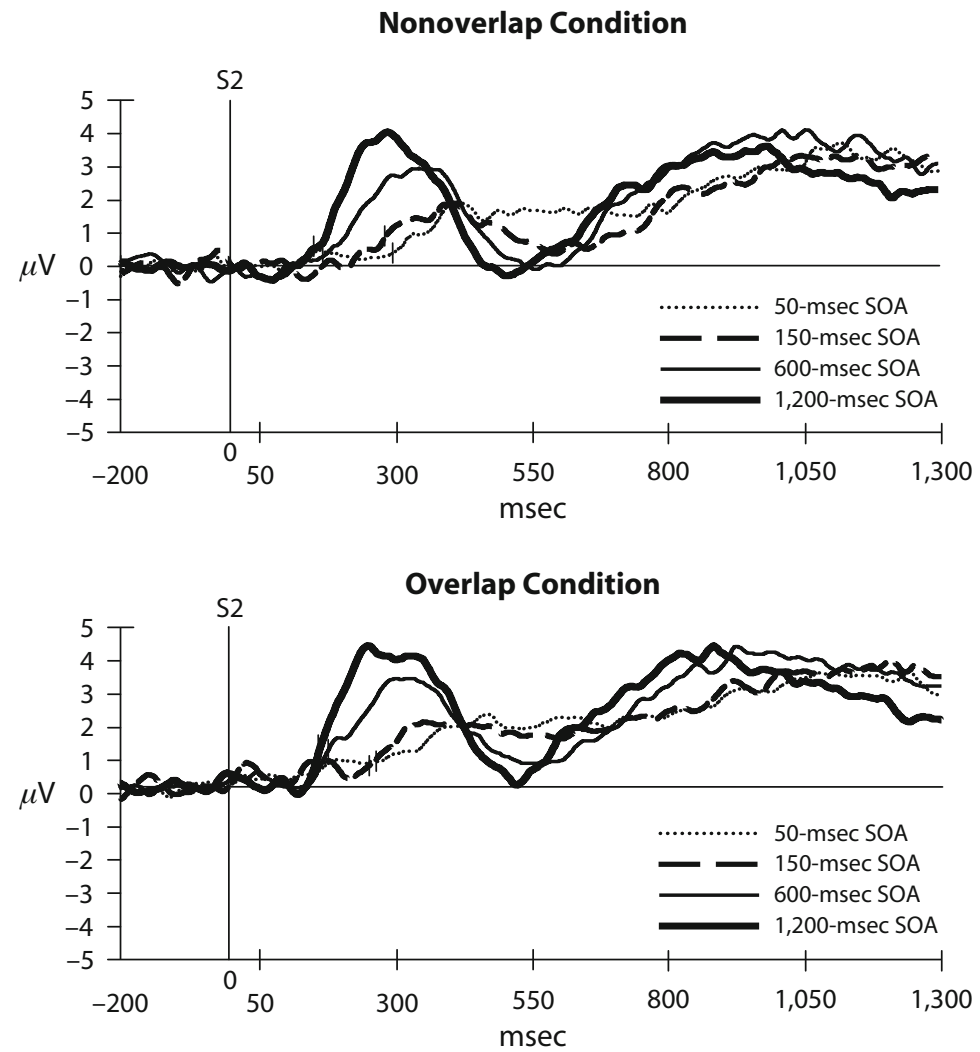

Figure 3. Task 2 stimulus (S2)-locked lateralized readiness potentials as a function of stimulus onset asynchrony (SOA) in the nonoverlap and overlap conditions. The small vertical line for each LRP condition indicates the average estimated LRP onset. (Negative voltages are plotted downward.) 
Table 3

Stimulus 2-Locked Lateralized Readiness Potential (S2-Locked LRP) Onsets, Response 2Locked Lateralized Readiness Potential (R2-Locked LRP) Onsets, and Response 1-Locked Lateralized Readiness Potential (R1-Locked LRP) Onsets on Task 2 for the Nonoverlap and Overlap Conditions As a Function of Stimulus Onset Asynchrony (SOA)

\begin{tabular}{|c|c|c|c|c|c|c|c|c|}
\hline \multirow[b]{3}{*}{ Condition } & \multicolumn{8}{|c|}{$\mathrm{SOA}(\mathrm{msec})$} \\
\hline & \multicolumn{2}{|c|}{50} & \multicolumn{2}{|c|}{150} & \multicolumn{2}{|c|}{600} & \multicolumn{2}{|c|}{1,200} \\
\hline & $M$ & $S E$ & $M$ & $S E$ & $M$ & $S E$ & $M$ & $S E$ \\
\hline \multicolumn{9}{|c|}{ S2-Locked LRP Onsets } \\
\hline Nonoverlap & 291.50 & 29.93 & 272.05 & 10.55 & 173.39 & 36.77 & 147.80 & 17.25 \\
\hline Overlap & 256.85 & 33.78 & 246.31 & 19.93 & 173.52 & 18.20 & 147.11 & 17.36 \\
\hline \multicolumn{9}{|c|}{ R2-Locked LRP Onsets } \\
\hline $\begin{array}{l}\text { Nonoverlap } \\
\text { Overlap }\end{array}$ & $\begin{array}{l}-320.86 \\
-353.37\end{array}$ & $\begin{array}{l}67.40 \\
50.57\end{array}$ & $\begin{array}{l}-283.08 \\
-298.05\end{array}$ & $\begin{array}{l}85.43 \\
35.63\end{array}$ & $\begin{array}{l}-221.24 \\
-220.36\end{array}$ & $\begin{array}{l}56.03 \\
84.10\end{array}$ & $\begin{array}{l}-193.89 \\
-194.39\end{array}$ & $\begin{array}{l}32.88 \\
50.60\end{array}$ \\
\hline \multicolumn{9}{|c|}{ R1-Locked LRP Onsets } \\
\hline Nonoverlap & -133.89 & 32.67 & -65.29 & 41.63 & 232.65 & 51.00 & 725.14 & 29.95 \\
\hline Overlap & -172.64 & 40.05 & -53.42 & 16.44 & 149.89 & 55.74 & 724.50 & 73.13 \\
\hline
\end{tabular}

onset at short SOAs rules out completely parallel central processing, but (as discussed below) does not rule out partially parallel central processing. Also, LRP onset was significantly different for the overlap $(205.95 \mathrm{msec})$ and nonoverlap $(221.18 \mathrm{msec})$ conditions $[F(1,9)=23.67$, $\left.M S_{\mathrm{e}}=196.19, p<.001\right]$. The interaction of task relationship and SOA was significant $\left[F(3,27)=3.42, M S_{\mathrm{e}}=\right.$ $456.43, p<.05]$. Follow-up analyses revealed that the latencies were significantly shorter for the overlap condition than the nonoverlap condition at both the $50-\mathrm{msec}$ SOA (difference $=34.65 \mathrm{msec}$ ) and the $150-\mathrm{msec}$ SOA $(25.74 \mathrm{msec})\left[F_{\mathrm{s}}(3,27) \geq 13.14, M S_{\mathrm{e}} \mathrm{s} \leq 329.76, p \mathrm{~s}<\right.$ $.01]$, but not at the $600-\mathrm{msec}$ SOA $(-0.13 \mathrm{msec})$ or the 1,200-msec SOA (0.69 msec).

R2-locked LRP. In the R2-locked analyses, each trial included a total EEG segment of 1,500 msec, starting from 1,000 msec preceding R2 and continuing until $500 \mathrm{msec}$ after. The waveforms were adjusted by subtracting the average voltage during a baseline interval 1,000 to $750 \mathrm{msec}$ before R2. We then followed the same steps used in the S2-locked analyses.

The resulting R2-locked LRP waveforms are shown in Figure 4, and the estimated LRP onsets latencies are shown in Table 3 . If Task 2 central operations are completely postponed by the central bottleneck at short SOAs, and if the LRP reflects response activation/motor preparation, then R2-locked LRP intervals should be similar for all SOAs. Contrary to this prediction, the effect of SOA was significant $\left[F(3,27)=30.44, M S_{\mathrm{e}}=2,793.33, p<\right.$ .001]. R2-locked LRP intervals were $106.37 \mathrm{msec}$ longer, on average, at the two shortest SOAs than at the two longest SOAs $\left[F(1,9)=53.29, M S_{\mathrm{e}}=4,246.37, p<.001\right]$. Neither task relationship nor its interaction with SOA was significant $\left(F_{\mathrm{s}}<1.0\right)$.

\section{Alternative LRP Analyses: R1-Locked LRP}

Although the observed increase in the R2-locked LRP interval at short SOAs is consistent with parallel central processing, it is also consistent with serial central pro- cessing. The R2-locked LRP interval reflects the duration of all processes following LRP onset, including response initiation and execution. Any prolongation of these processes at short SOAs would increase the R2-locked LRP interval (just as we observed) even if central operations were performed strictly serially. A special case of such prolongation, noted above, is a response initiation bottleneck. Another possibility is that, after the Task 1 response has been made, participants begin preparing both hands to make a rapid Task 2 response. Short SOAs would allow less preparation time, prolonging response processes and lengthening the R2-locked LRP interval.

The S2-locked analysis is also not definitive. Although strictly serial central processing clearly predicts an effect of SOA on S2-locked LRP onset, parallel central processing need not predict the absence of SOA effects. Responses might be activated in parallel, but less rapidly at short SOAs than at long SOAs (e.g., due to capacity sharing).

To definitively assess parallel central processing, a new analysis is required. Our solution was to time-lock Task 2 LRP to R1. If central operations are strictly serial, then the Task 2 LRP should begin only upon completion of Task 1 central operations, an event closely time-locked to R1 (differing only in response initiation time). Thus, time-locked to R1, the LRP should start at a similar point in time for the 50- and 150-msec SOAs. Parallel central processing, however, predicts an earlier LRP onset at the 50 - than at the 150-msec SOA. This is because, relative to R1, S2 appears earlier at the 50-msec SOA than at the 150msec SOA, giving R2 activation a 100-msec head start.

Figure 5 shows Task 2 LRP time-locked to R1, and Table 3 shows the estimated R1-locked latencies. Consistent with parallel central processing, the LRP started much earlier at the 50-msec SOA than at the $150-\mathrm{msec}$ SOA for both the nonoverlap condition $(69 \mathrm{msec})[F(1,9)=$ $\left.19.41, M S_{\mathrm{e}}=1,212.39, p<.01\right]$ and the overlap condition $(119 \mathrm{msec})\left[F(1,9)=65.37, M S_{\mathrm{e}}=1,087.20, p<\right.$ $.001]$. These R1-locked LRP data argue convincingly that central processing is parallel rather than strictly serial. 


\section{Nonoverlap Condition}

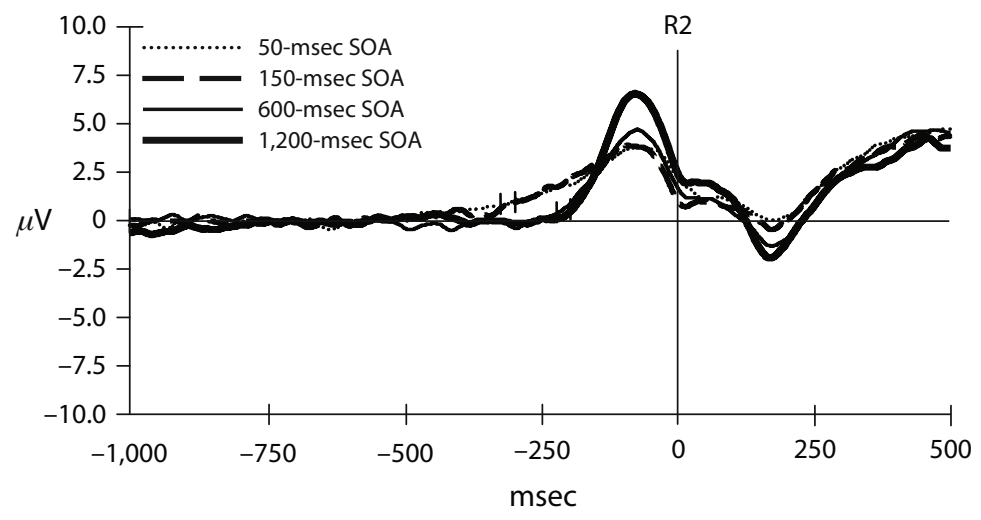

Overlap Condition

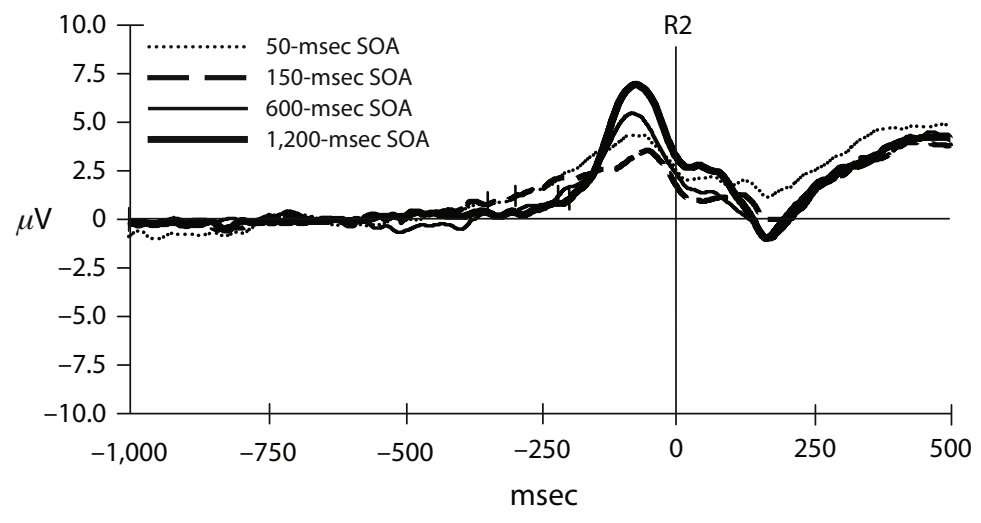

Figure 4. Task 2 response (R2)-locked lateralized readiness potentials as a function of stimulus onset asynchrony (SOA) in the nonoverlap and overlap conditions. The small vertical line for each LRP condition indicates the average estimated LRP onset. (Negative voltages are plotted downward.)

\section{DISCUSSION}

The present study investigated whether response activation can occur in parallel for two tasks. We used both LRP and backward correspondence effects as converging operations, while minimizing the potential for response conflicts. In addition, we examined whether parallel central processing is limited to task pairs with dimensional overlap.

The behavioral data from the S1-R2 dimensional overlap condition showed a backward correspondence effect, consistent with parallel central processing. The LRP data converged on the same conclusion. R1-locked LRP to Task 2 started 119 msec earlier at the 50-msec SOA than at the 150-msec SOA, indicating that Task 2 response activation began while Task 1 central processing was still under way. Similar LRP results were found in the nonoverlap condition. Apparently, strong dimensional overlap is not necessary to produce parallel central processing (although, as discussed below, it might enhance it).

In summary, whereas previous measurements of LRPs and backward correspondence (in separate studies) suggested opposite conclusions regarding parallel central pro- cessing, these measures converged on the same conclusion in the present study. It is worth asking why our LRP results differ from those of Osman and Moore (1993). Although the R2-locked LRPs in their Experiment 1 showed a trend consistent with parallel central processing, the trend was nonsignificant and numerically much smaller than the effects reported here. One possible explanation is that our use of manual and vocal responses minimized response conflicts, making it easier for participants to perform central operations in parallel (see Ruthruff et al., 2001).

\section{Implications for Theories of Dual-Task Performance}

Although the R2-locked LRPs suggest that Task 2 response activation occurs in parallel with Task 1 central operations, the S2-locked LRPs show that the response activation was weak. The early LRP amplitude at short SOAs was only about $10 \%-20 \%$ of that at long SOAs (see Figure 3). Should one be impressed by the modest "leakage" of Task 2 central operations during the bottleneck delay or impressed by the strong attenuation $(80 \%-90 \%)$ ?

At first blush, parallel central processing might appear to rule out the central bottleneck model. As noted above, 


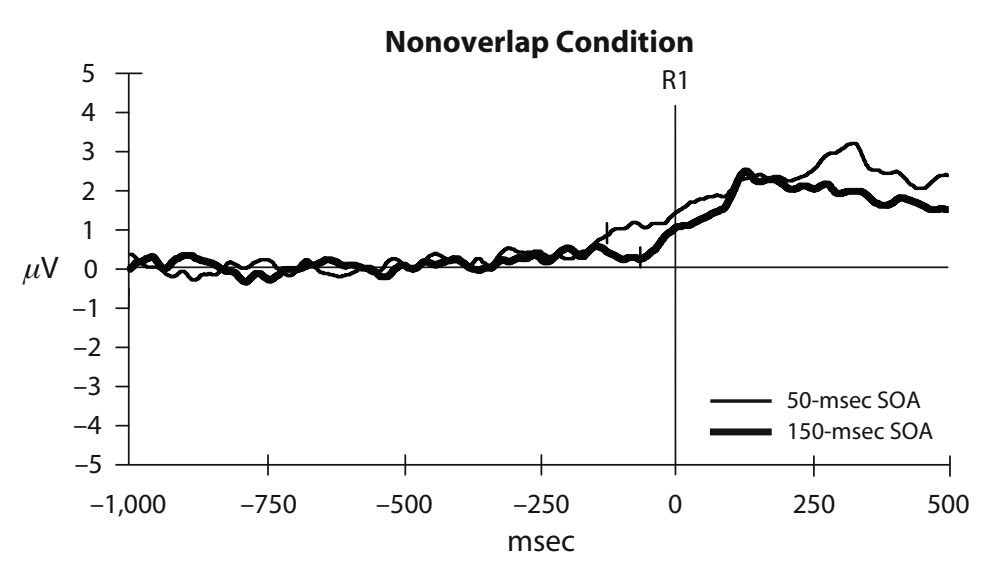

Overlap Condition

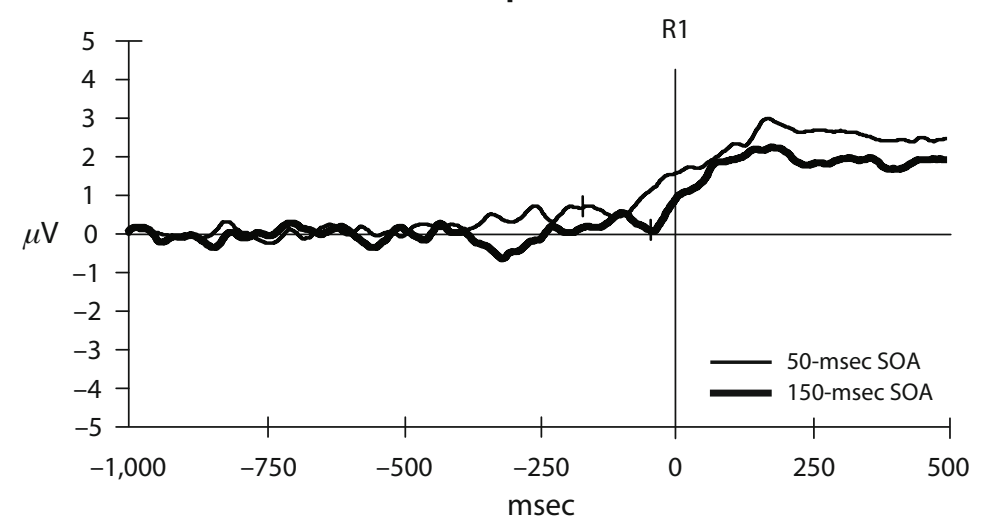

Figure 5. Task 1 response (R1)-locked lateralized readiness potentials on Task 2 at the 50-msec and 150-msec stimulus onset asynchronies (SOAs) in the nonoverlap and overlap conditions. The small vertical line for each LRP condition indicates the average estimated LRP onset. (Negative voltages are plotted downward.)

however, Task 2 central processing was strongly attenuated at short SOAs. Furthermore, logically speaking, a central bottleneck does not necessarily imply the absence of parallel response activation. While selecting the Task 1 response, a single-channel mechanism might accidentally execute the Task 2 mapping rules as well (to some degree), causing modest activation of the Task 2 response. Alternatively, there may be some automatic response activation (not dependent on any central resources). The key point is that the brain might be unable to select more than one response at a time, yet still able to simultaneously activate multiple responses to multiple stimuli.

Consistent with this hypothesis, we found no evidence of progress in selecting R2 during Task 1 central processing. Despite some parallel central processing, RT2 was still slowed dramatically, by more than $400 \mathrm{msec}$, at short SOAs. This observation causes us to question whether parallel central processing serves any useful function (at this level of practice) or instead reflects a failure of selective task activation.

A failure of selective task activation is most likely with dimensional overlap between tasks. When both tasks use the same stimuli or responses, activation of one task might inadvertently activate the other task as well. Indeed, the S2-locked LRP at the two shortest SOAs started $30 \mathrm{msec}$ earlier, on average, with dimensional overlap than without. Note that, because mean RT1 was actually $34 \mathrm{msec}$ longer in the overlap condition, strictly serial central processing should have caused the Task 2 LRP to onset later in this condition, producing the opposite result.

\section{Conclusions}

Previous backward correspondence studies and LRP studies have yielded opposite conclusions regarding parallel central processing. After combining both measures in the same study (the dimensional overlap condition), we found that they converged on the same conclusion: Central processes can operate in parallel. The key difference between our study and previous LRP studies might be that we minimized response conflicts by combining a vocal and a manual response. Parallel response activation occurred even without dimensional overlap, although to a lesser degree. We argue that dimensional overlap may increase the likelihood that activation of one task inadvertently activates the other task. 


\section{AUTHOR NOTE}

This research was supported by the College of Liberal Arts at Oregon State University, NASA Grant NCC 2-1325, and Grant NSC92-2413-H194-023 from the National Science Council of Taiwan. Correspondence concerning this article should be addressed to M.-C. Lien, Department of Psychology, Oregon State University, Corvallis, OR 97331-5303 (e-mail: mei.lien@oregonstate.edu).

\section{REFERENCES}

Coles, M. G. H. (1989). Modern mind-brain reading: Psychophysiology, physiology, and cognition. Psychophysiology, 26, 251-269.

EIMER, M. (1998). The lateralized readiness potential as an on-line measure of central response activation processes. Behavior Research Methods, Instruments, \& Computers, 30, 146-156.

Hommel, B. (1998). Automatic stimulus-response translation in dualtask performance. Journal of Experimental Psychology: Human Perception \& Performance, 24, 1368-1384.

Kornblum, S., \& LeE, J.-W. (1995). Stimulus-response compatibility with relevant and irrelevant stimulus dimensions that do and do not overlap with the response. Journal of Experimental Psychology: Human Perception \& Performance, 21, 855-875.

LiEn, M.-C., \& Proctor, R. W. (2000). Multiple spatial correspondence effects on dual-task performance. Journal of Experimental Psychology: Human Perception \& Performance, 26, 1260-1280.

Lien, M.-C., \& Proctor, R. W. (2002). Stimulus-response compatibility and psychological refractory period effects: Implications for response selection. Psychonomic Bulletin \& Review, 9, 212-238.

Logan, G. D., \& Schulkind, M. D. (2000). Parallel memory retrieval in dual-task situations: I. Semantic memory. Journal of Experimental Psychology: Human Perception \& Performance, 26, 1072-1090.
Mordkoff, J. T., \& Gianaros, P. J. (2000). Detecting the onset of the lateralized readiness potential: A comparison of available methods of procedures. Psychophysiology, 37, 347-360.

Osman, A., \& Moore, C. M. (1993). The locus of dual-task interference: Psychological refractory effects on movement-related brain potentials. Journal of Experimental Psychology: Human Perception \& Performance, 19, 1292-1312.

PASHLER, H. (1984). Processing stages in overlapping tasks: Evidence for a central bottleneck. Journal of Experimental Psychology: Human Perception \& Performance, 10, 358-377.

Ruthruff, E., Johnston, J. C., \& Van Selst, M. (2001). Why practice reduces dual-task interference. Journal of Experimental Psychology: Human Perception \& Performance, 27, 3-21.

Sangals, J., Roß, L., \& Sommer, W. (2004). Time pressure effects on information processing in overlapping tasks: Evidence from the lateralized readiness potential. Acta Psychologica, 117, 275-294.

Sommer, W., Leuthold, H., \& Schubert, T. (2001). Multiple bottlenecks in information processing? An electrophysiological examination. Psychonomic Bulletin \& Review, 8, 81-88.

Tombu, M., \& Jolicceur, P. (2005, July). Assessing output interference after extensive practice. Poster presented at the 15th Annual Meeting of the Canadian Society for Brain, Behaviour and Cognitive Science, Université de Montréal, Canada.

WELFORD, A. T. (1952). The "psychological refractory period" and the timing of high-speed performance-A review and a theory. British Journal of Psychology, 43, 2-19.

(Manuscript received October 3, 2005; revision accepted for publication May 30, 2006.) 